\title{
HIV transmission risk during breast feeding was greatest during the early months of life
}

Miotti PG, Taha TE, Kumwenda NI, et al. HIV transmission through breastfeeding: a study in Malawi.JAMA 1999 Aug 25;282:744-9.

QUESTION: In HIV positive mothers, what is the risk for HIV infection in their breast feeding infants?

Design

Cohort study with 24 month follow up.

\section{Setting}

A tertiary care hospital in Blantyre, Malawi.

\section{Participants}

672 infants who were born to HIV infected women who had not received antiretroviral drugs during pregnancy or after childbirth. Infants had to be HIV negative on the first postnatal visit at 6 weeks of age, be breast fed, and have a second follow up visit.

\section{Assessment of risk factors}

Time of infant HIV infection through breast feeding was taken to be the midpoint between the last postnatal negative polymerase chain reaction (PCR) test result and the first postnatal positive PCR test result. Time at risk for infant HIV infection through breast feeding was the interval between the first postnatal negative PCR test result and the first postnatal positive PCR test result. Potential risk factors assessed included mother's age, maternal symptoms of HIV or death within 2 years of giving birth, parity, delivery mode, breast problems (painful swelling, other signs of infection, or cracked or bloody nipples), and infant's birthweight.

\section{Main outcome measure}

HIV infection in infants determined by PCR testing. Both HIV antibody (enzyme linked immunosorbent assay) testing and immunoblotting were used to confirm HIV infection in infants $\geqslant 15$ months of age.

\section{Main results}

47 infants (7\%) became infected with HIV through breast feeding. The cumulative risk for infection after 5 , 11,17 , and 23 months was $3.5 \%, 7.0 \%, 8.9 \%$, and $10.3 \%$, respectively. The HIV infection incidence rates per person month were $0.7 \%$ during months 1 to 5 and decreased to $0.2 \%$ during months 18 to 23 ( $\mathrm{p}=0.01$ for trend). Univariate analysis showed that the only risk factor associated with HIV transmission was low parity; higher parity was protective $(<4 v \geqslant 4$ infants; relative risk [RR] $0.4,95 \%$ CI 0.2 to 0.9 ). The greatest risk was to second born infants. Using multivariate analysis, the protective association of higher parity was maintained (RR 0.2, CI 0.09 to 0.6 ), and older maternal age ( $\geqslant 25 \mathrm{y}$ ) was also protective (RR 0.4, CI 0.2 to 0.8 ).

\section{Conclusions}

In HIV positive mothers, the risk for HIV transmission to their breast feeding infants was greatest during 1 to 5 months of age. Higher parity and older maternal age were associated with a lower risk for transmission.

\section{COMMENTARY}

The prospective cohort study by Miotti and colleagues evaluated the risk for HIV transmission from breast feeding mothers to infants who were negative for HIV DNA at their first 6 week postnatal visit. The most important finding in this study was that the risk for transmission was highest during the first 6 months of life; high parity and older maternal age were protective. The lack of data about transmission during the first 6 weeks is unfortunate because immaturity of the newborn immune system and increased cell counts in early breast milk may facilitate HIV transmission. The study setting did not allow the authors to evaluate such other potentially important risk factors as gestational age at birth and maternal immunological and virological status.

The World Health Organisation (WHO) and the Join United Nations Programme on HIV/AIDS (UNAIDS) estimate that an HIV positive mother has a 15\% risk for transmitting HIV to her breast fed child. ${ }^{1}$ The risk for HIV transmission may be particularly high in resource poor areas where HIV testing is not available and little access to safe breast feeding alternatives exists. ${ }^{2}$ The recent WHO, United Nations Children's Fund (UNICEF), and UNAIDS statement incorporates recommendations for HIV positive mothers. ${ }^{1}$ Options include use of commercial formulas; wet nursing; use of heat treated, expressed breast milk; or exclusive breast feeding with abrupt early weaning. Further study of these issues and subsequent public health recommendations will be challenged by global variations in breast feeding practices, cultural beliefs, and economics.

Clay Triplehorn, DO Family Practice Residency Program Bangor, Maine, USA Kimberly A Moody, PhD, RN-CS, ANP University of Southern Maine Portland, Maine, USA

1 Joint policy statement on HIV and infant feeding: WHO, UNICEF, UNAIDS Statement on Current Status of WHO UNAIDS/UNICEF Policy Guidelines. Geneva, September 1999.

2 Fowler MG, Bertolli J, Nieburg P. When is breastfeeding not best? The dilemma facing HIV-infected women in resourcepoor settings. JAMA 1999;282:781-3. 